\title{
New saurichthyid actinopterygian fishes from the Anisian (Middle Triassic) of southwestern China
}

Wu Feixiang, Sun Yuanlin, Xu Guanghui, Hao Weicheng, Jiang Dayong, and Sun Zuoyu Acta Palaeontologica Polonica 56 (3), 2011: 581-614 doi: http://dx.doi.org/10.4202/app.2010.0007

A new genus Sinosaurichthys of the Saurichthyidae with three new species, S. longipectoralis, $S$. longimedialis, and S. minuta, are described and compared with Saurichthys. The new genus is represented by more than a hundred almost complete skeletons, collected from the strata corresponding to the Upper Member of the Guanling Formation (Pelsonian, Anisian, Middle Triassic) of two localities: Yangjuan of Panxian County, Guizhou Province, and Dawazi of Luoping, Yunnan Province, China. Sinosaurichthys differs from Saurichthys in having an unusual dermal pectoral girdle, high insertion of pectoral fin, relatively dorsally positioned axial skeleton in the abdominal region, and in the absence of branchiostegal rays. These differences are supposed to reflect the different life styles between the two genera. Sinosaurichthys, like the extant needlefish, probably has a better ability for cruising in surface water than Saurichthys . In addition, these species of Sinosaurichthys are compared, and their morphological differences also probably reflect individual adaptations for different habitats at the two localities.

Key words: Actinopterygii, Saurichthyidae, Sinosaurichthys, Anisian, Triassic, Guizhou, Yunnan, China.

Wu Feixiang [wufeixiang@ivpp.ac.cn] and Xu Guanghui [xuguanghui@ivpp.ac.cn], Key Laboratory of Orogenic Belts and Crustal Evolution, School of Earth and Space Sciences, Peking University, Beijing 100871, China, and Key Laboratory of Evolutionary Systematics of Vertebrates, Institute of Vertebrate Paleontology and Paleoanthropology, Chinese Academy of Sciences, Beijing 100044; Sun Yuanlin, [ylsun@pku.edu.cn ] corresponding author, Hao Weicheng [whao@ pku.edu.cn], Jiang Dayong [djiang@pku.edu.cn ], and Sun Zuoyu [sunzuoyu@ pku.edu.cn], Key Laboratory of Orogenic Belts and Crustal Evolution, School of Earth and Space Sciences, Peking University, Beijing 100871, China.

This is an open-access article distributed under the terms of the Creative Commons 
Attribution License (for details please see creativecommons.org), which permits unrestricted use, distribution, and reproduction in any medium, provided the original author and source are credited.

Fof Full text $(5.322 .4 \mathrm{kB})$ 